Brief Communication

\section{Observation of telepsychiatry service in a teaching hospital of eastern Nepal during COVID-19 pandemic}

\author{
Dhana Ratna Shakya* \\ Professor, Department of Psychiatry, BP Koirala Institute of Health Sciences, Dharan, Nepal
}

\begin{abstract}
Considering the geographical complexity and adversity, online communication and consultation are viable method in Nepal. The COVID-19 pandemic has accelerated the already starting trend of use of these technologies in medicine. In BPKIHS, telemedicine efforts were already initiated; lockdown rather warranted its maximum use. Here is an account of the observation made in telepsychiatry service provided by a consultant psychiatrist of its department of psychiatry. It is an institute-based observation noted for all the telepsychiatry consultations in 9 random duty days of the COVID-19 pandemic. Basic necessary information was noted down in a semi-structured proforma, like: socio-demographic, clinical information and advice provided.

There were 104 subjects; 73 follow-up and 31 new: 60 male and 44 female cases. Clients of multi-ethnic groups were the most from urban, then semi-urban and least from rural areas. More consultations were for young age-groups and from nearby districts of Sunsari. Mood, somatic (sleep), anxiety were the top presenting complaints and 8/104 clients had suicidal symptoms. Maximum follow-up cases were improving. The most common diagnoses were: Mood (Depression and Bipolar), Anxiety, Psychosis and Substance use disorders. Most common treatment advices included: Antidepressants, Antipsychotics, Benzodiazepines and Counseling/psycho-education. Telepsychiatry is a viable method of delivering service even during the pandemic.
\end{abstract}

\section{More Information}

*Address for Correspondence: Dr. Dhana Ratna Shakya, Professor, Department of Psychiatry, B.P. Koirala Institute of Health Sciences, Dharan, Nepal, Email: drdhanashakya@yahoo.com

Submitted: October 05, 2021

Approved: October 13, 2021

Published: October 14, 2021

How to cite this article: Shakya DR. Observation of telepsychiatry service in a teaching hospital of eastern Nepal during COVID-19 pandemic. Insights Depress Anxiety. 2021; 5: 025-028.

DOI: 10.29328/journal.ida.1001027

ORCiD: orcid.org/0000-0002-3796-5426

Copyright: (c) 2021 Shakya DR. This is an open access article distributed under the Creative Commons Attribution License, which permits unrestricted use, distribution, and reproduction in any medium, provided the original work is properly cited.

Check for updates

OPEN ACCESS

\section{Nature of COVID-19 pandemic}

The molecular, epidemiological and clinical features of SARS-CoV-2 have been gradually known. So far, it is evident that the nature of Corona virus disease 2019 (COVID-19) infection (mainly high transmission, mutation to new strains and consequent higher transmission and pathogenicity) resulted in rapid worldwide spread, morbidity and mortality [1]. Subsequent circumstances globally urged for a comprehensive approach with the balance of: protection of health, prevention of economic and social disruption, and respect of human rights [2].

\section{Need of the hour}

Some lessons learnt from the pandemic are general and others are local context based. Along with evolving facts about the virus and disease, the management strategies and policies are also forthcoming. The need of the hour, clear so far is breaking the chain of the infection through SMS IPC (Social distancing, mask, sanitization and infection prevention and control) measures and some local manners/customs like 'Namaste' in place of touching ways of greeting, intimacy or warmth [3]. In such a state of International Concern of Public Health, all including consumers (patients, students), providers (professionals, teachers, service institutes, government) and society (public) should bear extraordinary responsibility for the health, and prevention of the infection and other health issues [4]. Such a need of novelty, urgency and responsibility is there in health service setting and treatment strategies too $[5,6]$.

\section{Novel approach, policies and strategies}

The method with social distancing and less touch, contact and aerosol generation as far as possible on one hand and on the other with a greatest possible respect to clients' individual needs (e.g. comorbidity, risks like old age), human-rights and dignity, would be the preference during the pandemic [6]. Current scenario compelled for various new strategies, modification in ongoing methods and replacement of ineffective and useless ways with effective ones. This need 
was everywhere for all levels of prevention, i.e. primary prevention, treatment, rehabilitation. Novelty is warranted in service, research, academia of health sciences and other areas of human life and society. Various concepts, models and modes are already underway in this line [5-9].

\section{Telepsychiatry: a novel strategy}

Helpline telephone consultation $[10,11]$. and Telemedicine/psychiatry services are novel methods [12]. Professional bodies have formulated guidelines regarding telemedicine [13] and telepsychiatry [14]. Wherever possible, available, useful and effective; these latest technology based innovations in treatment modality should receive the place and they are going to be increasing swiftly.

\section{Telepsychiatry in Nepal and BPKIHS}

Considering the geographical complexity and adversity, online communication and consultation, i.e. Telemedicine are viable method in Nepal. As in the country- Nepal [15], telemedicine efforts were already initiated in BPKIHS. The COVID-19 pandemic has accelerated the already starting trend of use of these technologies in medicine; lockdown rather warranted its maximum use [16].

BPKIHS started Helpline mobile phones for needy people to have free consultations with respective specialists when it closed OPDs in lockdown periods. This useful method was soon followed by Telemedicine/psychiatry service in the time of crisis and helplessness. In our telepsychiatry, we were providing service to $10-25$ patients per day, 3 days in a week. Through virtual platforms (e.g. Google meet, Zoom etc.), we are having frequent virtual meetings regarding clinical care and academic activities. Academic activities/online classes are still heavily based on the virtual platforms.

\section{Method and results}

\section{Telepsychiatry in our observation}

Here, the author intends to depict an account of the observation made in the telepsychiatry service. It is an institute-based observation noted by a consultant psychiatrist of its department of psychiatry for all the telepsychiatry consultations in 9 random duty days of the COVID-19 pandemic. Basic necessary information was noted down in a semi-structured proforma, including: socio-demographic, clinical information and advice provided.

There were 104 cases; 73 follow-up and 31 new: 60 male and 44 female. Clients of multi-ethnic groups (main being Brahmin, Rai, Newars and Tarai ethnic castes) were the most from urban, then semi-urban and the least from rural areas. More consultations were for young age-groups and from nearby districts, i.e. Sunsari, Morang, Jhapa, Siraha though there were also for other age-groups and from far places of other provinces (Table 1).

Mood, somatic (sleep), anxiety were the top presenting complaints and 8/104 clients had suicidal symptoms. Maximum follow-up cases were improving, a few had ongoing either static or worsening symptoms (Figure 1).

The most common diagnoses were: Mood (Depression and Bipolar), Anxiety, Psychosis and Substance use disorders. Most common treatment advices included: Antidepressants, Antipsychotics, Benzodiazepines and Counseling/psychoeducation (Table 2).

\section{Discussion and conclusion}

\section{Reflection to our telepsychiatry observation}

More Nepalese citizens live in villages. Geographic adversities, lack of roads and transport services, poverty and ignorance about the services along with minimal government priority and policy are some important reasons behind the huge mental health gap in Nepal. Access to the mental health services is still far from satisfaction in developing countries. These countries including Nepal struggle hard to provide even

\begin{tabular}{|c|c|c|c|c|c|c|c|}
\hline Category & S. No. & Variables & No. $(\%)$ & Category & S. No. & Variables & No. $(\%)$ \\
\hline \multirow{2}{*}{ Case type } & 1 & New & $31(29.81)$ & \multirow{7}{*}{ Age group } & 1 & $11-20$ & $13(12.5)$ \\
\hline & 2 & Follow up & $73(70.19)$ & & 2 & $21-30$ & $32(30.77)$ \\
\hline \multirow{2}{*}{ Gender } & 1 & Female & $44(42.31)$ & & 3 & $31-40$ & $26(25.00)$ \\
\hline & 2 & Male & $60(57.69)$ & & 4 & $41-50$ & $13(12.5)$ \\
\hline \multirow{11}{*}{ Caste/ Ethnic group } & 1 & Brahmin & $30(28.85)$ & & 5 & $51-60$ & $16(15.39)$ \\
\hline & 2 & Chhetri & $8(7.69)$ & & 6 & $61-70$ & $3(2.89)$ \\
\hline & 3 & Newar & $15(14.42)$ & & 7 & $\geq 71$ & $1(0.96)$ \\
\hline & 4 & Rai & $22(21.15)$ & \multirow{10}{*}{ District } & 1 & Sunsari & 60 (57.69) \\
\hline & 5 & Tamang & $3(2.89)$ & & 2 & Morang & $13(12.5)$ \\
\hline & 6 & Gurung & $3(2.89)$ & & 3 & Jhapa & $10(9.62)$ \\
\hline & 7 & Terai ethnic & $15(14.42)$ & & 4 & Siraha & $8(7.69)$ \\
\hline & 8 & Dalit & $4(3.85)$ & & 5 & Dhanusha & $3(2.89)$ \\
\hline & 9 & Muslim & $2(1.92)$ & & 6 & Bhojpur & $2(1.92)$ \\
\hline & 10 & Magar & $1(0.96)$ & & 7 & Kavre & $1(0.96)$ \\
\hline & 11 & Limbu & $1(0.96)$ & & 8 & Dhankutta & $4(3.85)$ \\
\hline \multirow{3}{*}{ Residence } & 1 & Village & $8(7.69)$ & & 9 & Saptari & $2(1.92)$ \\
\hline & 2 & Semi-urban & $29(27.89)$ & & 10 & Bharatpur & $1(0.96)$ \\
\hline & 3 & City & $67(64.42)$ & & & Total subjects & $104(100.00)$ \\
\hline
\end{tabular}




\begin{tabular}{|c|c|c|c|c|c|c|c|}
\hline Category & S. No. & Variables & No. $(\%)$ & Category & S. No. & Variables & No. (\%) \\
\hline \multirow{11}{*}{ Diagnosis } & 1. & Organic mental & $4(3.85)$ & \multirow{11}{*}{ Diagnosis } & 5. & Anxiety & $31(29.81)$ \\
\hline & 1.1 & SD & $2(1.92)$ & & 5.1. & Panic & $7(6.73)$ \\
\hline & 1.2 & Headache & $1(0.96)$ & & 5.2. & OCD & $4(3.85)$ \\
\hline & 2. & SUD & $5(4.81)$ & & 6. & Stress related & $2(1.92)$ \\
\hline & 2.1 & AUD & $4(3.85)$ & & 7.1 & Dissociative & $1(0.96)$ \\
\hline & 2.2 & Nicotine/ other & $4(3.85)$ & & 7.2 & Somatoform & $2(1.92)$ \\
\hline & 3. & F20 & $11(10.58)$ & & 8. & Mental retardation & $1(0.96)$ \\
\hline & 4. & Mood & $69(66.35)$ & & 9. & Drug side effect & $2(1.92)$ \\
\hline & 4.1 & Depression & $52(50.00)$ & & 10. & DSH & $8(7.69)$ \\
\hline & 4.2 & BPAD & $15(14.42)$ & & 11. & Other & $2(1.92)$ \\
\hline & 4.3 & Other/Dysthymia & $2(1.92)$ & & 12. & Not adequate & $1(0.96)$ \\
\hline \multirow{6}{*}{ Management } & 1. & Antipsychotic & 48. $(46.15)$ & \multirow{6}{*}{ Management } & 7. & Thyroid & $2(1.92)$ \\
\hline & 2. & $\begin{array}{l}\text { TCA, SSRI, MAOI, other } \\
\text { anti-depressant }\end{array}$ & 75 (72.12) & & 8. & Vitamin supplement & $5(4.81)$ \\
\hline & 3. & Bzp, hypnotic & $42(40.39)$ & & 9. & Other & $10(9.62)$ \\
\hline & 4. & Lithium & $9(8.65)$ & & 10. & Psychological: Education & $23(22.12)$ \\
\hline & 5. & $\mathrm{NaV} / \mathrm{CBZ}, \mathrm{AE}$ & $9(8.65)$ & & 11. & Refer & $1(0.96)$ \\
\hline & 6. & THP & $1(0.96)$ & & 12. & Bring in ER/OP & $4(3.85)$ \\
\hline
\end{tabular}

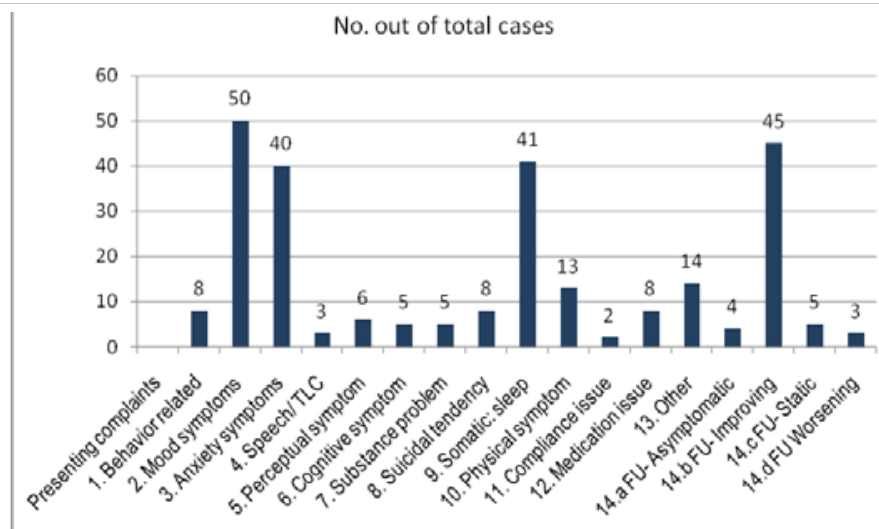

Figure 1: Presenting complaints in Telepsychiatry.

essential and basic, primary health care; even more during the pandemic. Nepal is a developing country with inadequate system, resource and monitoring mechanism; and was grossly unprepared for such a massive mayhem of the pandemic. There are new cases, emerging issues, with old cases of various health issues including mental, neurological and substance use (MNS) disorders. These clinical profiles of presenting complaints and diagnoses comply largely with the pictures seen in help line service of same setting $[10,11]$. Many with reactive states (sub-syndromal, less severe but distressing conditions like stress, stress situation), stress related disorders, mild depression etc. abound during the pandemic. Magnitude and burden of COVID per se is overwhelming. On the other hand, other health issues were not less which were somehow sidelined during the pandemic.

Many needy people were afraid of going to service while people with minor issues were exposed unnecessarily to the risk of COVID infection as reported in general in this tertiary care hospital set up [16]. Service facility, provider institutes, organizations, spread of information and adequate communication on part of the state/government and awareness, alert steps, help seeking through appropriate modes including novel methods (e.g. helplines, telemedicine) on part of clients would add to the better outcome in the pandemic as indicated by the literature of this short period. These novel strategies e.g. helplines, telemedicine/psychiatry were much talked off, discussed, and the protocols were developed throughout, e.g. Nepal (Nepal Medical Council) protocol for telephone or online consultations- telemedicine/ telepsychiatry.

Our observation too replicates that Telepsychiatry is a viable method of delivering service even during the pandemic (to address new and follow-up psychiatric cases). Hence, it should be extended and promoted in all the way possible for all the time.

\section{References}

1. Hu B, Guo H, Zhou P, Shi Z. Characteristics of SARS- CoV-2 and COVID-19. Nature Reviews. Microbiology. 2021; 19: 141-154. PubMed: https://pubmed.ncbi.nIm.nih.gov/33024307/

2. Yang M, Li H, Sun J, Zhao Y, Tang D. Focus on Characteristics of COVID-19 with the Special Reference to the Impact of COVID-19 on the Urogenital System. Curr Urol. 2020; 14: 79- 84

PubMed: https://pubmed.ncbi.nlm.nih.gov/32774232/

3. Shakya DR, Thapa SB, Kar SK, Sharma V, Uchida N, et.al. COVID-19 across Countries: Situation and Lessons for Pandemic control. J BPKIHS. 2020; 3: 39-49.

4. Shakya DR, Shrestha RR, Koirala S, Upadhyaya Kafle S, Subed $P$, et.al. Social responsibility for health during COVID-19 pandemic. JBPKIHS. 2021; 4: 48-55.

5. Xiao C. A Novel Approach of Consultation on 2019 Novel Coronavirus (COVID-19)-Related Psychological and Mental Problems: Structured Letter Therapy. Psychiatry Investig. 2020; 17: 175-176. PubMed: https://pubmed.ncbi.nlm.nih.gov/32093461/

6. Vishak Acharya V, Unnikrishnan B. A novel approach to combat COVID-19 - A risk stratification model with FAIR intervention. J Healthc Qual Res. 2021; 36: 55-56.

PubMed: https://pubmed.ncbi.nlm.nih.gov/33342757/

7. Cag Y, Icten S, Isik-Goren B, Baysal NB, Bektas B, et al. A nove approach to managing COVID-19 patients; results of lopinavir plus doxycycline cohort. Eur J Clin Microbiol Infect Dis. 2021; 40: 407-411. PubMed: https://pubmed.ncbi.nlm.nih.gov/32856202/ 
8. Yadav AR, Mohite SK. A Novel approach for treatment of COVID-19 with Convalescent plasma. Res J Pharmaceuit Dosage, Forms Technol. 2020; 12: 227-230.

9. Sparks RSJ, Aspinall WP, Brooks-Pollock E, Cooke RM, Danon L, et al 2021 A novel approach for evaluating contact patterns and risk mitigation strategies for COVID-19 in English primary schools with application of structured expert judgement. R Soc Open Sci. 2021; 8: 201566. PubMed: https://pubmed.ncbi.nIm.nih.gov/33614088/

10. Shakya DR. Observation and Lesson from Psychiatry Help-Line of a Teaching Hospital in Eastern Nepal during COVID-19 Pandemic Lockdown. Clin Med. 2020; 2: 1021.

11. Shakya DR. Problems shared in Psychiatry help-line of a Teaching hospital in eastern Nepal during COVID-19 Pandemic Lockdown. Insights in Depress Anxiety. 2020; 4: 037-039.

PubMed: https://www.heighpubs.org/hda/ida-aid1017.php
12. Bains J, Greenwald PW, Mulcare MR, Leyden D, Kim J, et al. Utilizing Telemedicine in a Novel approach to COVID-19 management and Patient experience in the Emergency Department. Telemed J E Health. 2021; 254-260.

PubMed: https://pubmed.ncbi.nlm.nih.gov/32821027/

13. Nepal Medical Council. Telemedicine Guidelines for Registered Medical Practitioners in Nepal. 2020.

14. Indian Psychiatry Society, Telemedicine Society of India, NIMHANS. Telepsychiatry Operational Guidelines. 2020.

15. Bhatta R, Aryal K, Ellingsen G. Oppertunities and Challenges of a Rural Tele-medicine Program in Nepal. J Nepal Health Res Counc. 2015; 13: 149-153.

PubMed: https://pubmed.ncbi.nIm.nih.gov/26744201/

16. Shakya DR, Mishra DR, Gyawali R, Rimal SP, Lama S, et.al. COVID-19 pandemic and BPKIHS: our Situation, Endeavors and Future direction. J BPKIHS. 2020; 3: 39-49. 